\title{
Reducing Dropout among Traumatized Alcohol Patients in Detoxification Treatment: A Pilot Intervention Study
}

\author{
Michael Odenwald ${ }^{a}$ Peter Semrau ${ }^{b}$ \\ ${ }^{a}$ University of Konstanz and ${ }^{b}$ Center of Psychiatry Reichenau, Germany
}

\author{
Key Words \\ Alcohol - Alcohol-addicted patients - Detoxification . \\ Retention/dropout - Traumatic life events · Posttraumatic \\ stress disorder - Psychoeducation - Group psychotherapy • \\ Treatment motivation - Group-control study
}

\begin{abstract}
Dropout rates from detoxification treatment are high. We tested whether high trauma event load was related to a higher dropout from alcohol detoxification. Furthermore, we studied the feasibility and effects of a short psychoeducational tool to increase retention among traumatized alcohol in-patients. Retention and treatment length were compared between treatment as usual (TAU) and standard therapy plus a psychoeducational group intervention on alcohol drinking related to stress and trauma (PAST). Patients with high trauma load were identified with the Trauma History Questionnaire. Of the 159 in-patients treated during the study period, 66 were included in the analysis: 33 in TAU and 33 in PAST. Sociodemographic characteristics did not differ between the groups. During TAU, patients with high trauma load tended to drop out more often $(p=0.056)$. Among patients with high trauma load, retention level increased from 29 to $80 \%(p=0.006)$, and among those with low trauma load
\end{abstract}

from 63 to $83 \%(p=0.250)$. Treatment length only tendentially improved among patients with lower burden $(p=$ 0.056). The pilot study supports the idea that detoxification treatment dropout occurs more often among alcohol patients with high trauma load and that their retention can be increased by a psychoeducational group intervention.

\section{Introduction}

Alcohol is one of the most harmful substances for both the individual and society [1]; billions are invested each year for therapy for its negative effects [2]. Detoxification is one of the most frequent forms of care for alcohol-dependent patients [3]. It is considered a crucial element that enables further engagement in a treatment network with multiple service providers who successively accompany patients through different parts of the rehabilitation process [4].

In Germany, alcohol detoxification usually takes place either in in-patient units of psychiatric hospitals or in general hospitals; in the latter, it is mostly focused on somatic withdrawal management and lasts a few days [5]. In psychiatric hospitals, 'qualified detoxification treatment' 
is offered, which comprises psychosocial and motivational elements for subsequent rehabilitative treatments in addition to somatic monitoring and management of withdrawal symptoms and complications; it takes up to 6 weeks to complete [4]. In 2010, 4,750 such beds were available on a national level [6]. Studies have clearly shown the superiority of qualified detoxification treatment over its purely somatic type $[5,7]$. In recent years, day clinic and out-patient models of qualified detoxification treatment have been emerging, showing effects at least as good as the in-patient programs $[8,9]$.

Dropout rates from alcohol detoxification are considered high in many countries, but prevalence rates are not known [10]. Indeed, retention in and dropout from alcohol detoxification treatment have rarely been studied [10], and the current understanding of the psychological processes involved remains rudimentary [10-12]. Dropout from alcohol detoxification was often quantified as discharge against medical advice or discharge in the first week of treatment [13].

In a UK study, $32.8 \%$ of 470 first admission alcohol patients dropped out of detoxification [12]. In a study from the USA, $28.5 \%$ of 1,454 drug and alcohol patients dropped out of detoxification [11]. In Scotland, dropout from different forms of alcohol treatment, including detoxification, exceeded 50\% [14]. In Germany, however, national statistics on dropout from alcohol detoxification are not available [15]. The data published by single institutions vary considerably. In one report, $20 \%$ of 529 consecutively admitted alcohol patients dropped out from in-patient detoxification [16]. In another clinic, it was $43 \%$ of 90 alcohol in-patients [10]. In an out-patient detoxification program, $10 \%$ of 141 alcohol patients dropped out [8]. In a 2007 national sample of patients from different German addiction treatment institutions (detoxification, long-term rehabilitation), discharge against medical advice occurred in $36.6 \%$ of alcohol patients [17].

The mode of ending detoxification treatment (regular discharge vs. dropout) is frequently considered an immediate outcome criterion of treatment success and predicts other positive outcomes [17]. Unfortunately, no such study exists that focuses on alcohol detoxification alone. Dropout from alcohol or drug detoxification was frequently related to subsequent relapse of abuse and readmission to detoxification treatments $[11,18]$, as well as failure to enroll in further substance use therapy [19]. In contrast, completion of detoxification was related to abstinence in a 1-month follow-up [19], and extended detoxification was related to higher rates of enrollment into further substance use therapies compared to 5 or less days of detoxification [20]. In Germany, one report followed up a large sample of alcohol detoxification patients (3-month relapse rate of 60.5\%) and showed that the group of patients with less than 7 days in treatment is at an especially high risk of relapse and repeated admission to detoxification compared to those with longer treatments [13].

Despite the need to develop specific interventions to reduce dropout in detoxification units [12], few reports on such attempts exist $[21,22]$. Recently, service providers have come under increasing pressure to improve treatment retention [3].

For the understanding of psychological processes related to addiction treatment completion, comorbid psychiatric disorders need to be considered because of their frequent co-occurrence [23]. There are very few studies on the effects of psychopathology on retention in alcohol detoxification. One study found that psychiatrists' psychopathology ratings at treatment entry were higher among those who later dropped out [10]. The study cited above looked at 470 first admission patients and found that borderline and antisocial personality disorders were more frequent, and depressive disorders less frequent, among those who dropped out; no differences were found in relation to posttraumatic stress disorder (PTSD) or other anxiety disorders [12].

The effects of traumatic experiences, PTSD and other posttraumatic disorders on retention in alcohol detoxification treatment have not received systematic attention thus far. There is evidence, however, to justify studying this question. First of all, there is a large comorbidity of alcohol dependency among PTSD patients, e.g. ex-combatants [24], and from the general population [25]. Additionally, among patients in alcohol treatment units, a large proportion have experienced traumatic events or suffer from PTSD [26, 27]. Secondly, substance-dependent patients with PTSD are generally in a worse psychological state than those without PTSD [28]. Growing evidence supports an etiological link between PTSD and the subsequent development of substance use disorders [29]. Neuropharmacological studies clearly support the notion that alcohol is effective for self-medicating PTSD and that alcohol withdrawal can reactivate PTSD symptoms [30]. In the same line, a pathophysiological link between early experiences of stress in life, related hormonal system changes, and the development of subsequent substance use disorders has received empirical support [31]. Furthermore, recent studies identified PTSD as a risk factor for substance use relapse and poorer treatment outcome [32], whereas patients with double diagnosis receiving 
PTSD treatment had a better addiction-related long-term outcome than those who did not receive it [33].

To our knowledge, there is currently no study available examining retention in detoxification among traumatized alcohol patients.

This pilot study investigates two main research questions: (1) it studies whether traumatized alcohol patients drop out more frequently from detoxification; and (2) we tested the feasibility and evaluated the short-term outcomes of a brief psychoeducational group intervention. This intervention was developed to prevent dropout among traumatized alcohol patients within an in-patient detoxification unit. In this study, we used trauma event load as the moderator variable for dropout from detoxification as PTSD could not directly be assessed. We first hypothesized that in treatment as usual (TAU), dropout from detoxification would be higher among patients with high as compared to low trauma event load. Secondly, we assumed that our psychoeducational group intervention would specifically decrease dropout among patients with high trauma event load, but not among patients with low trauma event load. Finally, we expected that treatment duration would only increase in the group of patients with high trauma load.

\section{Methods}

\section{Design}

This quasi-experimental study was implemented in an alcohol detoxification unit of a public psychiatric hospital (with 29 inpatient beds) in southern Germany. The unit has approximately 800 admissions per year. In 2009, the average treatment length was 13.4 days (SD 10.2). In a controlled design, TAU and standard therapy plus a specific group intervention, PAST (psychoeducational group intervention on alcohol drinking related to stress and trauma), were compared in relation to their effect on treatment retention (primary outcome measure) and treatment length (secondary outcome measure). In order to test the hypothesized baseline differences between traumatized and nontraumatized patients, as well as the hypothesized specificity of the intervention, we recruited patients with high and low trauma event load in both treatment conditions. The study was implemented in one center, and in order to minimize contamination, treatment conditions were implemented sequentially. TAU was studied in month 1 ; PAST was implemented in month 3 . In month 2 , no data were assessed.

\section{Intervention}

TAU comprised standard medical, psychological, and nursing care; a daily 'morning round' with all patients $(30 \mathrm{~min})$ led by the medical doctor in charge; and a twice-weekly $(2 \times 45 \mathrm{~min})$ information session led by a social worker with about 4-8 participants. The information session served as an opportunity to ask questions mostly related to general aspects of subsequent therapy. Pa- tients were selected for this group autonomously by the nursing staff. Exclusion criteria were (1) severe current withdrawal symptoms, (2) severe cognitive and neuropsychological problems, (3) crisis intervention, and (4) severe comorbid psychiatric condition (e.g. psychosis, depression, and suicidality). Usually patients were allocated to this group after severe withdrawal symptoms had abated. Participation was voluntary. In the intervention condition, this information group was exchanged for manualized standardized group psychoeducation: PAST. The rest of the care package stayed the same, including the way that patients were allocated to this group and the voluntary participation. The nursing staff was not aware of the change of group contents. The social worker who led the groups was blinded to the selection of group members and subgroup membership (high or low trauma load). PAST is an open group intervention, consisting of four sessions ( $45 \mathrm{~min}$ ) that were held twice weekly, i.e. repetition of the program after 2 weeks. The aim of PAST is to give participants the opportunity to develop specific insights and exchange their experiences with the help of a supportive and directive trainer. The aim of the first session is to highlight the connection between the experience of stress and the use of alcohol. Session 2 introduces the distinction between 'stressful life event' and 'traumatic event' using the DSM-IV definitions, and informs the patients about typical PTSD symptoms. In session 3, the goal is to increase awareness of drinking alcohol as representing an attempt to cope with PTSD symptoms. In addition to this, the reactivation of PTSD symptoms through alcohol withdrawal is also emphasized. In session 4, ways out of this vicious circle are discussed on the basis of patients' experiences as well as treatment possibilities.

PAST was implemented without complications to the routine of the detoxification unit. During the project, no adverse incidents occurred, e.g. severe psychiatric decompensation.

\section{Measurements}

On the day of admission or soon after, nursing staff members informed the patient about the study. They received written information and a consent form to declare their voluntary study participation. If they agreed to participate, nursing staff delivered questionnaires to the patient and asked them to complete them. Completed questionnaires were returned to the nursing staff. The questionnaires comprised sociodemographic questions and the German version of the Trauma History Questionnaire (THQ) [34, 35]. The THQ is a frequently used list of 24 stressful and traumatic event types, such as being exposed to violent assault, accident, rape, etc. For each event type, the respondent indicates whether he/she has experienced it during his/her lifetime and if so, at which age he/she first experienced it. The stability of the self-reported THQ items was confirmed by a testretest study [34]. In this study, based on the information regarding how many of the THQ event types have ever been experienced, we computed a sum score (range: 0-24). In our study, this 24-item THQ event list achieved a satisfactory internal consistency (Cronbach's $\alpha: 0.80$ ). The most frequent event types were news about the illness or death of a close person (73\%); seeing dead bodies (48\%); experiencing a severe accident (45\%); being confronted with somebody severely injured or killed (45\%); and experiencing fear of death in another situation (41\%). In this study we used the THQ sum score as a moderator variable for treatment dropout. This is justified as the number of experienced traumatic events has been shown to be one of the best predictors 
Fig. 1. Flow chart of recruitment and inclusion of study participants.

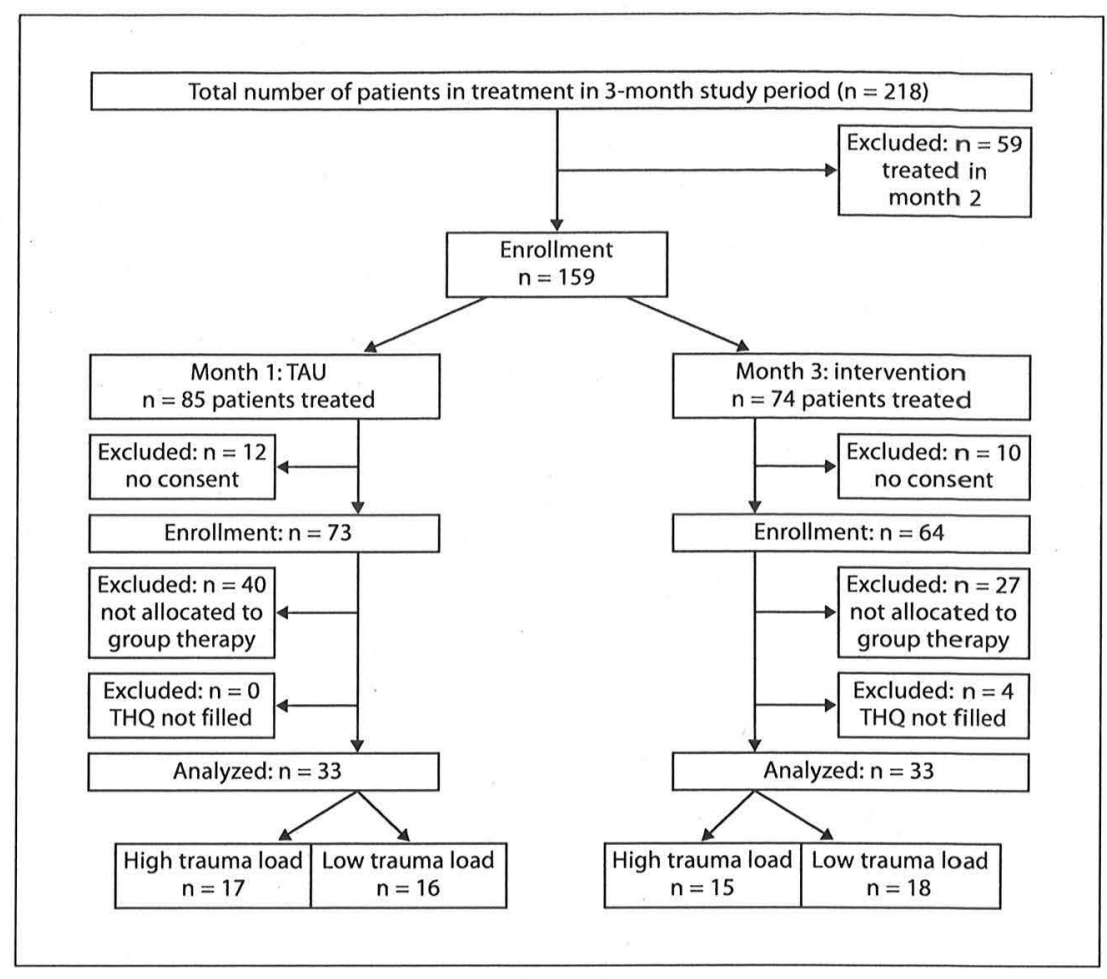

of PTSD and other posttraumatic psychopathologies in the sense of a dose-effect relationship [36]. Outcome variables were circumstances of discharge, as recorded in the patients' hospital file and length of treatment. Circumstances of discharge were the following: 'against medical advice', 'disciplinary', 'regular', and 'transferred to subsequent treatment'. The latter referred to psychotherapy units within or outside the clinic. The two latter circumstances of discharge were considered treatment completion, while the first two were considered dropouts.

\section{Participants}

In total, 159 patients were in treatment during study months 1 and 3. 137 gave their consent to be included in the study. During the two study months, 70 patients took part in at least one group session and of these, 66 of them completed the THQ. Participation in group therapy was possible for all patients irrespective of their consent to the study participation. Of this final sample, 33 took part in TAU and 33 in PAST (fig. 1). The proportion of patients included in the study in months 1 and 3 did not differ $(\mathrm{p}=0.49)$.

Based on the sum score of THQ, we used a median-split to separate respondents with high and low load of traumatic experiences ( $<6$ events vs. $\geq 6$ ). In TAU, 17 respondents were highly burdened by traumatic events and 16 were not. In PAST, 15 were highly burdened and 18 were not. Table 1 shows the characteristics of the groups. The proportion of male and female patients as well as the age of participants and the sum of THQ events were comparable in TAU and PAST ( $\mathrm{p}=0.792,0.231,0.305$ ), and did not differ between trauma load subgroups across conditions.

\section{Ethical Approval}

The University of Konstanz Research Review and Ethics Board approved the study.

\section{Data Analysis}

Data were analyzed using SPSS version 19 for Mac and R (package survival). We report means, SD, and percentages. Due to the small sample size, we relied on Fisher's exact test where preconditions of $x^{2}$ tests were not fulfilled when comparing percentages. Group differences of quantitative variables were tested using ANOVA, Student's t test, or a Wilcoxon test, the latter where preconditions were not fulfilled. First order error probability was set to 0.05 . For multiple tests, we used a Bonferroni correction. This leads to a corrected two-sided $p$ value of 0.025 . Equality of variances between patients with high and low trauma load was tested using Levene's test.

Using Kaplan-Meier survival analysis and a log-rank test, we tested whether the survival function of both treatment conditions and of participants with high and low trauma load differed. Dropout was defined as a critical event and regular completion as a censored event.

We tested the influence of different predictor variables on treatment time until dropout using Cox regression models. Here, we also defined regular discharge as a censored event. In three nested models, we successively entered more predictor variables and their interactions. In model 1 , we entered age and gender; in model 2, the sum score of the THQ and treatment condition. In model 3 , we additionally entered the interaction term between 
Table 1. Sociodemographic and other characteristics of participants

\begin{tabular}{|c|c|c|c|c|c|c|c|c|c|c|}
\hline & \multirow[t]{2}{*}{ All $(n=66)$} & \multicolumn{4}{|l|}{ TAU } & \multicolumn{4}{|l|}{ PAST } & \multirow[t]{2}{*}{$p^{1}$} \\
\hline & & all $(n=33)$ & $\begin{array}{l}\text { high trauma } \\
\text { load }(n=17)\end{array}$ & $\begin{array}{l}\text { low trauma } \\
\text { load }(n=16)\end{array}$ & $p^{2}$ & all $(n=33)$ & $\begin{array}{l}\text { high trauma } \\
\text { load }(n=15)\end{array}$ & $\begin{array}{l}\text { low trauma } \\
\text { load }(n=18)\end{array}$ & $\mathrm{p}^{2}$ & \\
\hline Average age, mean (SD) & $44.0(10.8)$ & $42.5(10.1)$ & $42.9(11.4)$ & $42.0(8.8)$ & 0.806 & $45.7(11.4)$ & $46.4(8.8)$ & $45.2(13.5)$ & 0.780 & 0.231 \\
\hline Female, \% (n) & $31.8(21)$ & $33.3(11)$ & $41.2(7)$ & $25.0(4)$ & 0.325 & $30.3(10)$ & $33.3(5)$ & $27.8(5)$ & 1.000 & 0.792 \\
\hline Average THQ sum, mean (SD) & $6.0(4.0)$ & $6.5(4.2)$ & $9.7(3.4)$ & $3.1(1.5)$ & $<0.001$ & $5.5(3.9)$ & $8.7(3.3)$ & $2.8(1.7)$ & $<0.001$ & 0.305 \\
\hline $\begin{array}{l}\text { Average days in treatment, } \\
\text { mean (SD) }\end{array}$ & $16.0(11.3)$ & $14.4(10.9)$ & $17.2(13.5)$ & $11.4(6.5)$ & 0.200 & $17.6(11.7)$ & $20.1(16.3)$ & $15.4(5.3)$ & 0.870 & 0.258 \\
\hline Completed treatment, \% (n) & $63.6(42)$ & $45.5(15)$ & $29.4(5)$ & $62.5(10)$ & 0.056 & $81.8(27)$ & $80.0(12)$ & $83.3(15)$ & 1.000 & 0.002 \\
\hline
\end{tabular}

${ }^{1}$ Two-sided general comparison of TAU and PAST. ${ }^{2}$ Two-sided comparisons of burdened and nonburdened participants within the two conditions.

THQ and treatment condition. We used successive likelihood ratio tests to assess the goodness of fit of the next more complex model against the prior model.

We report correlations between the variables of interest, i.e. Pearson correlations between continuous variables, point-biserial correlations between binary and continuous variables and Phi between binary variables.

\section{Results}

The sum of THQ events and the frequency of single THQ events, as well as age of first event and average age of all events, did not differ between the two treatment conditions. Thirteen of 24 events were more frequently reported in the high trauma condition: fear of death (67 vs. $16 \%$; $\mathrm{p}<0.001)$, robbery $(58$ vs. $9 \%$; $\mathrm{p}<0.001)$, witness of injury or killing ( 68 vs. $22 \%$; $p<0.001$ ), armed assault (46 vs. $3 \%$; $p<0.001$ ), violent robbery (49 vs. $6 \%$; $p<$ 0.001 ), private body parts being touched against one's will ( 42 vs. $3 \%$; $\mathrm{p}<0.001$ ), experiencing severe damage (39 vs. $3 \% ; \mathrm{p}<0.001$ ), learning about the unexpected death of somebody close ( 91 vs. $56 \% ; \mathrm{p}=0.001$ ), being a victim of housebreaking while not at home ( 36 vs. $3 \% ; \mathrm{p}=0.001$ ), assault without a weapon ( 33 vs. $3 \% ; p=0.002$ ), being seriously beaten within the family ( 49 vs. $19 \% ; \mathrm{p}=0.011$ ), forced sex/rape ( 30 vs. $3 \% ; \mathrm{p}=0.003$ ), and being exposed to a man-made disaster ( 27 vs. $6 \% ; \mathrm{p}=0.024$ ).

Patients with regular and irregular discharge from care tended to differ in relation to the number of THQ events [irregular vs. regular: mean $=7.2$ (SD 4.6) vs. 5.3 (3.6); $\mathrm{p}=0.066$. This difference was more pronounced in the TAU condition [7.8 (4.5) vs. $5.0(3.6) ; \mathrm{p}=0.058$ ] than in the trauma psychoeducation condition [5.5 (4.8) vs. 5.5 (3.7); $\mathrm{p}=0.805]$. Two event types were more frequent among patients with irregular discharge from care: 'being victim of housebreaking while not at home' (38 vs. $10 \% ; p=0.010)$ and 'private body parts being touched against one's will' (38 vs. 14\%; $\mathrm{p}=0.030$ ). The category for the rest ('any other stressful event') was more frequent among patients with regular discharge ( 8.3 vs. $33 \%$; $\mathrm{p}=$ 0.023).

Of the 66 participants, 42 successfully completed detoxification treatment (64\%) and 24 dropped out. Testing the first hypothesis, regular release occurred in 15 of 33 individuals (45.5\%) during TAU; highly burdened individuals tended to drop out more frequently: 10 among the low burdened (62.5\%) and 5 among the highly burdened patients $(29.4 \% ; \mathrm{p}=0.056$; table 1$)$. Comparing all patients with a high burden of traumatic events with all those who had a low burden also showed a trend for significance, i.e. that the former dropped out of treatment more frequently $(p=0.085)$. In contrast, 27 of 33 patients in PAST (81.8\%) completed treatment and no difference occurred between groups: 15 of the 17 low burdened (83.3\%) and 12 of the $15(80 \%)$ highly burdened patients completed detoxification $(\mathrm{p}=1.000)$.

In PAST, significantly more patients completed detoxification treatment compared to TAU (table $1 ; \mathrm{p}=0.002$ ). When testing our second hypothesis, highly burdened individuals dropped out significantly less in the PAST condition compared to TAU $(\mathrm{p}=0.006)$. Among low burdened individuals, no statistical significant differences occurred between treatment conditions $(p=0.250$; fig. 2a).

The distribution of days in detoxification treatment showed a clear difference between all participants with high and low trauma load ( $\mathrm{p}=0.001$; fig. $2 \mathrm{~b}$ ). Cases with extremely high and low treatment duration occurred 
Fig. 2. Effects of trauma group psychoeducation (PAST) on treatment effects among alcohol patients in detoxification treatment with high and low trauma load. a Retention in detoxification treatment; bars represent percentages of patients who completed in-patient detoxification. b Days in detoxification treatment; bars represent means (SD). Numbers below bars represent number of patients in subgroups.

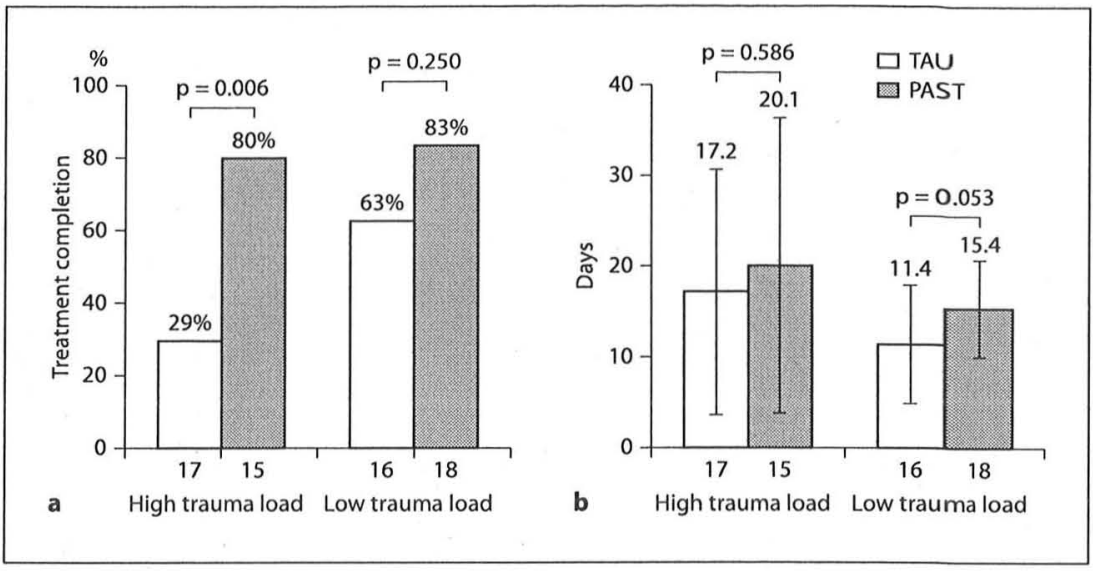

only in the former group ( 5 cases with $>30$ days, 2 cases with $<4$ days). The equality of variances was also not given for patients with high and low traumatic burden in TAU and PAST ( $\mathrm{p}=0.032$ and 0.007 ).

Average treatment length did not differ between conditions (table $1 ; \mathrm{p}=0.258$ ) nor between all patients with low and high trauma load [mean $=18.6$ (SD 14.7) vs. 13.5 (6.1); $\mathrm{p}=0.386]$. Testing our third hypothesis by comparing patients with high or low trauma load across conditions, we found no difference between the highly burdened patients $(\mathrm{p}=0.586$; fig. $2 \mathrm{~b}$ ). However, patients with low trauma load had a statistical trend for more days in treatment in PAST than in TAU $(p=0.056)$. Neither the TAU nor the PAST groups showed a statistically significant difference between highly and low burdened patients $(\mathrm{p}=0.200$ and 0.870 ; table 1$)$.

We computed Kaplan-Meier survival curves for both treatment groups (fig. 3). The survival curves for both treatment conditions differed significantly $(\mathrm{p}=0.003)$, showing that time in treatment until dropout was greater in the trauma psychoeducation group. Comparing survival curves of patients with high and low trauma load, we found no difference $(\mathrm{p}=0.660)$.

We developed Cox regression models to predict treatment dropout. In three nested models, we tested whether the inclusion of treatment condition and trauma load (model 2) beyond age and gender (model 1) as well as the interaction term (model 3) increased goodness of fit. The correlations ranged from low to moderate between treatment retention and treatment condition as well as trauma load $(0.378, p=0.002 ;-0.227, p=0.066)$. Predictor variables were not correlated with each other $(r<0.2, p\rangle$ $0.15)$. Goodness of fit of model 1 did not improve com-

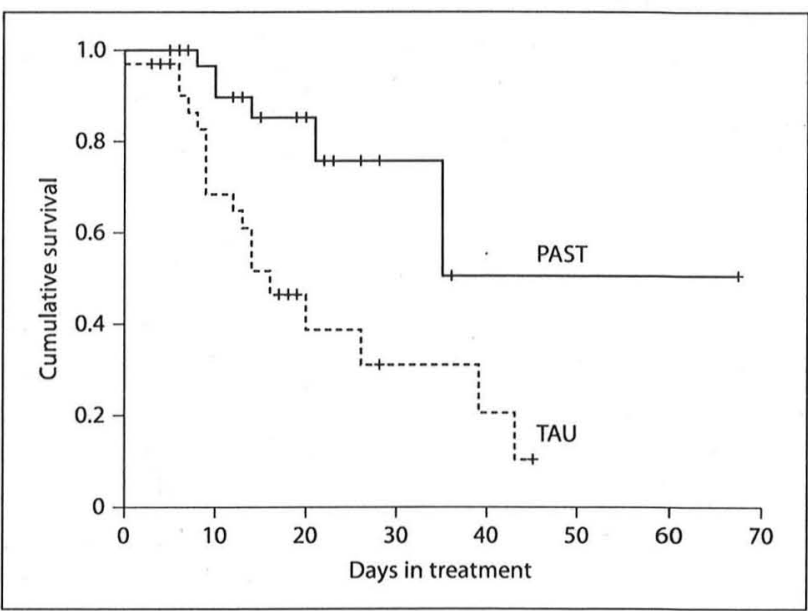

Fig. 3. Survival functions of the two studied groups, TAU and PAST.

pared to the beginning block (beginning block: $-2 \mathrm{LL}=$ 152.529; model 1: $-2 \mathrm{LL}=151.146 ; \chi^{2}=1.383 ; \mathrm{p}=0.501$ ). None of the predictors reached significance [OR (age): 1.003, 95\% CI: 0.965-1.043, p = 0.888; OR (gender): 0.588, 95\% CI: $0.246-1.402, \mathrm{p}=0.231$ ]. Model 2 brought a significant improvement compared to model 1 ( $-2 \mathrm{LL}=$ $\left.144.059 ; \chi^{2}=7.087 ; \mathrm{p}=0.029\right)$, but only treatment condition significantly contributed to a better model fit [OR (treatment condition): $0.312,95 \%$ CI: $0.116-0.836, \mathrm{p}=$ 0.021; OR (THQ sum): 1.022, 95\% CI: 0.879-1.188, p = 0.779]. Model 3, which included the two-way interaction between treatment condition and THQ sum, did not improve the fit of the model $\left[-2 \mathrm{LL}=144.055 ; \chi^{2}=0.004\right.$; 
$\mathrm{p}=0.951 ;$ OR (interaction): 1.010, 95\% CI: 0.737-1.385, $\mathrm{p}=0.951]$. Based only on the variables in model 2 , our final model had a significant improvement of model fit $\left(-2 L L=145.348 ; \chi^{2}=7.994 ; p=0.018\right)$ and included a significant contribution of treatment condition (OR: 0.306, 95\% CI: 0.117-0.800, $\mathrm{p}=0.016$ ) and a nonsignificant contribution of THQ sum (OR: 1.039, 95\% CI: 0.900-1.200, $\mathrm{p}=0.602)$.

\section{Discussion}

The interpretation of the reported data on treatment retention needs to be made with caution; the rates of retention and dropout cannot be treated as prevalence rates because they are based on a selected subgroup of all alcohol detoxification patients treated in our clinic during the study period ( $42 \%$ for the whole study period and $39 \%$ during TAU).

The results of our study support our hypothesis that dropout from standard alcohol detoxification is higher among patients with high trauma event load as compared to patients with low event load. Based on this small number of patients, the reported difference shows a substantial effect, although only achieving tendential statistical significance.

The reported results also provide the first empirical evidence for the idea that the completion rate of detoxification treatment for alcohol patients can be increased by a psychoeducational group intervention that focuses on stress, trauma, and drinking. In this study, the reported effect cannot be explained by potential sociodemographic and trauma-related a priori group differences. This result argues strongly for the further study of PAST and other attempts to increase retention in alcohol detoxification.

We could not clearly demonstrate, however, the hypothesized specificity of our intervention: although dropout decreased more among patients with high trauma event load, it also showed a decrease in the low burdened group. While this latter effect was not statistically significant in our study, by increasing the sample size, the effect could have been demonstrated. In sum, it remains unclear whether the reported intervention effect is due to specific or unspecific mechanisms, i.e. whether detoxification treatment completion among alcohol patients burdened by traumatic experiences can be enhanced by a specific trauma psychoeducation tool or whether such a tool can increase therapy motivation among all patients. The unspecific effect of PAST is supported by a signifi- cant increase of treatment length among patients with low traumatic burden in PAST and the results of our survival analysis.

The third hypothesis, i.e. intervention increases the treatment length among patients highly burdened by traumatic events but not among patients with low event load, can be rejected. Surprisingly, we found a trend for an increased treatment length only in the low burdened group. The drastic increase in treatment completion among patients highly burdened by traumatic experiences was not accompanied by an increase in treatment length, as one might intuitively expect. The reasons for this discrepancy are currently not clear. In our opinion, this could in part be explained by statistical effects, such as a ceiling effect; increasing treatment length above 20 days might simply become increasingly difficult due to the policies of health insurance funds. However, this contrasts with the high variation of treatment length in this group.

The high variability of days in detoxification treatment in the group with high traumatic burden might reflect the specific problems of this patient group. PTSD, other trauma-related disorders, and interpersonal problems are highly prevalent among cases with high trauma load [37]. Due to this high treatment need, substance abuse patients with comorbid disorders have longer treatments than those without them [38], which explains the extremely high treatment lengths in our study. At the same time, the group of highly burdened patients also shows severe interpersonal problems and hostility that can interfere with treatment retention, explaining early dropouts [39]. Furthermore, alcohol patients with high traumatic burden often show functional substance use patterns, i.e. alcohol use to cope with sleep problems, intrusive memories, or depression [32]. It is well known that alcohol in the short term diminishes PTSD-related symptoms, but alcohol withdrawal reactivates them [30]. This might also explain the high rate of early dropout among this group when comorbid PTSD is not adequately addressed. This mechanism of symptom reactivation might account for extended withdrawal symptoms and that cravings can be elicited by intrusive memories and vice versa [40]. It is important to note that the study took place in an acute ward, which might have provoked traumatic recollections in patients with trauma histories. In sum, we found some plausible hypotheses for extremely short and prolonged treatments, but future studies are needed to replicate and explain these results.

The survival curves support the idea that patients in PAST 'survived' longer, i.e. dropped out later from detoxification treatment than patients in TAU. The results of the 
Cox survival analysis show that the treatment condition, i.e. whether a patient received stress and trauma psychoeducation or not, was related to time until dropout. Stress and trauma psychoeducation significantly increased time until dropout, while our trauma burden score (THQ sum score) was not related to it nor were age and gender of the patient. Additionally, the interaction between treatment condition and trauma burden was not related to time until dropout. This might reflect the fact that PAST is of general interest to patients and increases emotional involvement regardless of trauma load. Participation and involvement in the therapy is generally believed to increase retention rates. Indeed, PAST provoked a high level of participation during group discussions among most participants. Further studies need to include adequate control groups to disentangle these effects, e:g. a third group with an unspecific training.

Our results suggest further ideas regarding potential mechanisms of psychoeducation on retention in detoxification treatment. We postulate that talking about past and current stressful and traumatic life experiences and the patients' coping attempts opens up the possibility for patients and therapists to talk more about the psychological problems in everyday life and subsequent treatment possibilities. We think that this had a positive influence on the therapeutic alliance, the patients' engagement in the treatment process, and the planning of subsequent treatment - all of which is known to predict treatment retention [41]. In future studies evaluating PAST, these variables need to be assessed in order to understand treatment mechanisms.

Our results put the current practice in many detoxification programs of deliberately not asking for traumatic experiences and PTSD into question. Drug abuse services have traditionally advocated a sequential treatment of substance abuse and other psychiatric disorders like PTSD in order to protect their clients during the stressful and emotionally overwhelming period of withdrawal symptoms. However, recently this basic assumption has been questioned [42]. It has been demonstrated that including trauma treatment in substance use treatment is safe and does not produce more adverse events [43], and that it has positive effects on the course of the substance use disorder [44]. Furthermore, it has been argued in other contexts that not asking about traumatic events might also have high costs for the individual and their treatment [45]. In sum, exploring stress and trauma during detoxification might represent a chance to uncover mechanisms maintaining addiction, increase motivation, and guide patients to the adequate treatments.
Our study has several limitations. First, the reported study was conducted with a selected subsample of patients receiving alcohol detoxification in our clinic. Reasons for this could relate to the particular patients who decided to take part and how they were allocated to the group therapy offered at the detoxification ward, e.g. patients with more severe alcohol-induced withdrawal symptoms and those with no treatment motivation might not have participated at all. Still, no systematic differences between the conditions in relation to allocation of patients occurred. Secondly, our assessment did not include measures of posttraumatic symptom load, PTSD, or other relevant psychopathology. In the study preparation phase, we found it difficult to convince the various clinicians responsible for the patients in our study that a detailed trauma assessment would not harm patients. The reported results support the idea that talking about traumatic stress does not lead to decompensation, but rather motivates patients to complete treatment. Thus, future studies should include a more detailed assessment of posttraumatic and other psychiatric symptoms. Thirdly, in the reported study, the systematic variation of some factors of influence was preliminary: the trainer and the center effects were not subjected to systematic variation. Given the fact that the number of treatment completers varies considerably between institutions [4], a multicenter approach would be needed to assess the effect of treatment centers. This would allow treatment conditions to be delivered in parallel instead of sequentially. In the reported study, sequential treatment delivery prevented the 'state of the art' randomization; therefore, it cannot be considered a true effect study. The study can also be criticized because of the restricted range of sociodemographic measures used to characterize individual study participants and of potential predictors of dropout, such as level of education. In previous studies, younger age predicted dropout [12]. In the reported study no such tendencies occurred and statistical methods confirmed that covariates seemed not to have influenced outcome measures. However, a broader range of potential predictors of dropout needs to be assessed in future studies. Further limitations include the small sample size and the limited range of outcome variables.

In future studies, beyond the suggestions made in the previous paragraphs, a second active treatment group should be included that is not thought to have a specific effect on traumatized patients, e.g. motivation enhancement therapy [22]. Attempts should be made to include a more representative sample of patients. Furthermore, a more complete set of outcome variables will need to be 
assessed, including a follow-up assessment on initiation of subsequent treatments, abstinence, and alcohol use. The proposed treatment could be extended beyond inpatient groups to out-patient settings as well as broadened to include patients with addictions to other types of drugs than alcohol.

In light of the high dropout rates in detoxification treatment and the importance of treatment completion for achieving better long-term outcomes, the reported result strongly encourages the further study of measures to improve retention rate in detoxification units among pa- tients burdened by traumatic experiences. Effective interventions to reduce dropout in detoxification have the potential to serve the health of patients while still operating within the financial constraints of the health ca re system.

\section{Acknowledgements}

We would like to thank Wolfgang Höcker, Brigitte Rockstroh, Markus Schnitzler and Thomas Elbert for their support and helpful comments. We thank James Moran for the English corrections.

\section{References}

1 van Amsterdam J, Opperhuizen A, Koeter $M$, van den Brink W: Ranking the harm of alcohol, tobacco and illicit drugs for the individual and the population. Eur Addict Res 2010;16:202-207.

2 Kraus L, Piontek D, Pabst A, Bühringer G Alcohol consumption, alcohol-related mortality, morbidity, social problems and costs in Germany (in German). Sucht 2011;57:119129.

3 Haley SJ, Dugosh KL, Lynch KG: Performance contracting to engage detoxificationonly patients into continued rehabilitation. J Subst Abuse Treat 2011;40:123-131.

4 Mann K: Current approaches in the therapy of the alcohol dependent; in Tölle R, Doppelfeld E (eds): Alcoholism: Identify and Treat (in German). Cologne, Deutscher Ärzteverlag, 2005.

5 Loeber S, Kiefer F, Wagner F, Mann K, Crois sant B: Treatment outcome after inpatient alcohol withdrawal: impact of motivational interventions: a comparative study (in German). Nervenarzt 2009;80:1085-1092.

6 Leune J: Versorgung abhängigkeitskranker Menschen in Deutschland; in Deutsche Hauptstelle gegen Suchtgefahren: Jahrbuch Sucht 2011. Geesthact, Neuland, 2011.

7 Stetter F, Mann K: The course of alcohol dependency after inpatient detoxification and motivation treatment. Nervenarzt 1997;68: 574-581.

8 Soyka M, Horak M, Morhart V, Moller HJ: The 'qualified outpatient detoxification' model project (in German). Nervenarzt 2001;72:565-569.

9 Weithmann G, Hoffmann M: A randomised clinical trial of in-patient versus combined day hospital treatment of alcoholism: primary and secondary outcome measures. Eur Addict Res 2005;11:197-203.
10 Braune NJ, Schroder J, Gruschka P, Daecke K, Pantel J: Determinants of unplanned discharge from in-patient drug and alcohol detoxification: a retrospective analysis of 239 admissions (in German). Fortschr Neurol Psychiatr 2008;76:217-224.

11 Callaghan RC, Cunningham JA: Gender differences in detoxification: predictors of completion and re-admission. J Subst Abuse Treat 2002;23:399-407.

12 Martinez-Raga J, Marshall EJ, Keaney F, Ball $\mathrm{D}$, Strang J: Unplanned versus planned discharges from in-patient alcohol detoxification: retrospective analysis of 470 first-episode admissions. Alcohol Alcohol 2002;37: 277-281.

13 Reker T, Richter D, Batz B, Luedtke U, Koritsch HD, Reymann G: Short-term effects of acute inpatient treatment of alcoholics. A prospective, multicenter evaluation study (in German). Nervenarzt 2004;75:234-241.

14 Newham R, Russell C, Davies JB: Planned and unplanned discharge from alcohol services in Scotland, 2004-2008. Alcohol Alcohol 2010;45:64-69.

15 Pfeiffer-Gerschel T, Kipke I, Flöter S, Karachaliou K, Lieb C, Raiser P: 2010 National Report (2009 data) to the EMCDDA by the Reitox National Focal Point. Munich, Deutsche Beobachtungsstelle für Drogen und Drogensucht, 2010.

16 Mann K, Stetter F: Die qualifizierte Entzugsbehandlung von Alkoholabhängigen: Entwicklung und Evaluation; in Mann K (ed): Neue Therapieansätze bei Alkoholproblemen. Lengerich, Pabst Science Publishers, 2002.

17 Hildebrand A, Sonntag D, Bauer C, Bühringer G: Treatment of substance-abuse patients in Germany: results from the 2007 statistical report on substance-abuse treatment facilities (in German). Sucht 2009;55:15-34.

18 Li X, Sun H, Marsh DC, Anis AH: Factors associated with seeking readmission among clients admitted to medical withdrawal management. Subst Abus 2008;29:65-72.
19 Rüesch P, Hättenschwiler J: Consequences of prelapse and treatment drop-out in inpatient drug detoxification: a one-month follow-up study (in German). Schweiz Arch Neurol Psychiatr 2002;153:238-244.

20 Booth BM, Blow FC, Cook CA, Bunn IY, Fortney JC: Relationship between inpatient alcoholism treatment and longitudinal changes in health care utilization. J Stud Alcohol 1997;58:625-637.

21 Katz EC, Brown BS, Schwartz RP, O'Grady KE, King SD, Gandhi D: Transitioning opioid-dependent patients from detoxification to long-term treatment: efficacy of intensive role induction. Drug Alcohol Depend 2011; 117:24-30.

22 Blondell RD, Frydrych LM, Jaanimagi U, Ashrafioun L, Homish GG, Foschio EM, Bashaw HL: A randomized trial of two be havioral interventions to improve outcomes following inpatient detoxification for alcohol dependence. J Addict Dis 2011;30:136148.

23 Conway KP, Compton W, Stinson FS, Grant BF: Lifetime comorbidity of DSM-IV mood and anxiety disorders and specific drug use disorders: results from the National Epidemiologic Survey on Alcohol and Related Conditions. J Clin Psychiatry 2006;67:247257.

24 Kulka RA, Schlenger WE, Fairbank JA, Hough RL, Jordan BK, Marmar CR, Weiss DS: Trauma and the Vietnam War generation: Report of Findings from the National Vietnam Veterans Readjustment Study. New York, Brunner/Mazel, 1990.

25 Kessler RC, Sonnega A, Bromet E, Hughes $\mathrm{M}$, Nelson CB: Posttraumatic stress disorder in the National Comorbidity Survey. Arch Gen Psychiatry 1995;52:1048-1060.

26 Dom G, De Wilde B, Hulstijn W, Sabbe B: Traumatic experiences and posttraumatic stress disorders: differences between treatment-seeking early- and late-onset alcoholic patients. Compr Psychiatry 2007;48:178185 
27 Driessen M, Schulte S, Luedecke C, Schaefer I, Sutmann F, Ohlmeier M, Kemper U, Koes ters G, Chodzinski C, Schneider U, Broese T Dette C, Havemann-Reinicke U: Trauma and PTSD in patients with alcohol, drug, or dual dependence: a multi-center study. Alcohol Clin Exp Res 2008;32:481-488.

28 Najavits LM, Harned MS, Gallop RJ, Butler SF, Barber JP, Thase ME, Crits-Christoph P Six-month treatment outcomes of cocainedependent patients with and without PTSD in a multisite national trial. J Stud Alcoho Drugs 2007;68:353-361.

29 Chilcoat HD, Breslau N: Posttraumatic stress disorder and drug disorders: testing causal pathways. Arch Gen Psychiatry 1998. 55:913-917.

30 Jacobsen LK, Southwick SM, Kosten TR: Substance use disorders in patients with posttraumatic stress disorder: a review of the literature. Am J Psychiatry 2001;158:11841190.

31 Schafer I, Teske L, Schulze-Thusing J, Homann K, Reimer J, Haasen C, Hissbach J, Wiedemann K: Impact of childhood trauma on hypothalamus-pituitary-adrenal axis activity in alcohol-dependent patients. Eur Ad dict Res 2010;16:108-114.

32 Waldrop AE, Back SE, Verduin ML, Brady KT: Triggers for cocaine and alcohol use in the presence and absence of posttraumatic stress disorder. Addict Behav 2007;32:634639.
33 Ouimette P, Moos RH, Finney JW: PTSD treatment and 5-year remission among patients with substance use and posttraumatic stress disorders. J Consult Clin Psychol 2003; 71:410-414

34 Green BL: Psychometric review of the Trauma History Questionnaire (self-report); in Stamm BH (ed): Measurement of Stress, Trauma and Adaptation. Lutherville, Sidran Press, 1996, pp 366-369.

35 Maercker A: Deutsche Übersetzung des Trauma History Questionnaire. Zürich, Universität Zürich, 2002.

36 Neuner F, Schauer M, Karunakara U, Klaschik C, Robert C, Elbert T: Psychological trauma and evidence for enhanced vulnerability for posttraumatic stress disorder through previous trauma among West Nile refugees. BMC Psychiatry 2004;4:34

37 Najavits LM, Schmitz M, Gotthardt S, Weiss RD: Seeking safety plus exposure therapy: an outcome study on dual diagnosis men. J Psychoactive Drugs 2005;37:425-435.

38 Bradley CJ, Zarkin GA: An inpatient profile of patients with a substance abuse diagnosis in Maryland. J Subst Abuse Treat 1997;14: 155-162.

39 Broome KM, Flynn PM, Simpson DD: Psychiatric comorbidity measures as predictors of retention in drug abuse treatment programs. Health Serv Res 1999;34:791-806.
40 Saladin ME, Drobes DJ, Coffey SF, Dansky BS, Brady KT, Kilpatrick DG: PTSD symp tom severity as a predictor of cue-elicited drug craving in victims of violent crime. Ad dict Behav 2003;28:1611-1629.

41 Meier PS, Donmall MC, McElduff P, Barrowclough C, Heller RF: The role of the early therapeutic alliance in predicting drug treat ment dropout. Drug Alcohol Depend 2006; 83:57-64.

42 Henslee AM, Coffey SF: Exposure therapy for posttraumatic stress disorder in a residential substance use treatment facility. Prof Psychol Res Pr 2010;41:34-40.

43 Killeen T, Hien D, Campbell A, Brown C Hansen $C$, Jiang $\mathrm{H}$, Kristman-Valente A Neuenfeldt C, Rocz-de la Luz N, Sampson R, Suarez-Morales L, Wells E, Brigham G Nunes E: Adverse events in an integrated trauma-focused intervention for women in community substance abuse treatment. Subst Abuse Treat 2008;35:304-311.

44 Hien DA, Campbell AN, Ruglass LM, Hu $\mathrm{MC}$, Killeen T: The role of alcohol misuse in PTSD outcomes for women in community treatment: a secondary analysis of NIDA's Women and Trauma Study. Drug Alcoho Depend 2010;111:114-119.

45 Becker-Blease KA, Freyd JJ: Research partic ipants telling the truth about their lives: the ethics of asking and not asking about abuse. Am Psychol 2006;61:218-226. 